\title{
E3 ubiquitin ligase Pirh2 enhances tumorigenic properties of human non-small cell lung carcinoma cells
}

\author{
Alexandra Daks ${ }^{1, *}$, Alexey Petukhov ${ }^{1,2,3, *}$, Olga Fedorova ${ }^{1}$, Oleg Shuvalov ${ }^{1}$, Valeriy \\ Merkulov' ${ }^{1}$, Elena Vasileva ${ }^{1}$, Alexey Antonov ${ }^{4}$, and Nikolai A. Barlev ${ }^{1}$ \\ ${ }^{1}$ Institute of Cytology, Russian Academy of Sciences, St Petersburg, Russia \\ ${ }^{2}$ Almazov Federal North-West Medical Research Centre, Institute of Hematology, St Petersburg, Russia \\ ${ }^{3}$ National Research University of Information Technologies, Mechanics and Optics, St Petersburg, Russia \\ ${ }^{4}$ MRC Toxicology Unit, Leicester, UK \\ * These authors made equal contribution \\ Correspondence to: Nikolai Barlev, email: nick.a.barlev@gmail.com
}

Alexandra Daks, email: alexandra.daks@gmail.com

Keywords: Pirh2, RCHY1, H1299, non-small cell lung cancer, drug resistance, EMT, xCELLigence

Received: October 07, $2016 \quad$ Accepted: November 07, 2016 Published: November 09, 2016

\section{ABSTRACT}

The product of RCHY1 human gene, Pirh2, is a RING-finger containing E3 ligase that modifies p53 with ubiquitin residues resulting in its subsequent degradation in proteasomes. Transcription of $\mathrm{RCHY1}$ is regulated by p53 itself thus forming a negative regulatory feedback loop. Functionally, by eliminating p53, Pirh2 facilitates tumorigenesis. However, the role of Pirh2 in cancer cells lacking p53 is yet not well understood. Therefore, we decided to elucidate the role of Pirh2 in p53-negative human non-small cell lung carcinoma cells, H1299. We found that ectopic expression of Pirh2 enhanced cell proliferation, resistance to doxorubicin, and increased migration potential. Ablation of Pirh2 by specific shRNA reversed these phenotypes. Mechanistically, Pirh2 increased mRNA and protein levels of the c-Myc oncogene. The bioinformatics data indicate that co-expression of both c-Myc and Pirh2 strongly correlated with poor survival of lung cancer patients. Collectively, our results suggest that Pirh2 can be considered as a potential pharmacological target for developing anticancer therapies to treat p53-negative cancers.

\section{INTRODUCTION}

Pirh2 was originally identified as an androgen receptor (AR) N-terminal-interacting protein (ARNIP) that exhibited ubiquitin ligase activity [1]. Later, Pirh2, in addition to Mdm2, was found to be one of the principal ubiquitin ligases that targets the major tumor suppressor p53 $[2,3]$.

P53 exerts its tumor suppressor functions as a transcription factor regulating expression of both coding and non-coding genes whose products induce cell cycle arrest and apoptosis in response to various forms of cellular stress $[4,5]$. Under normal conditions p53 is a short-lived protein, which undergoes rapid ubiquitindependent degradation in proteasomes [6]. However, upon genotoxic stress p53 becomes post-translationally modified by several covalent moieties, including phosphorylation, acetylation and methylation $[7,8]$.
Importantly, in response to DNA damage proteasomes themselves undergo covalent modifications that inactivate their proteolytic activity [9] resulting in further stabilization of p53. It was shown that Pirh2, in contrast to Mdm2, was able to ubiquitinate $\mathrm{p} 53$ phosphorylated on Ser15, one of the hallmark of p53 activation in response to DNA damage [10]. Thus, Pirh2 seems to be the major regulator of p53 under stress conditions [3]. Yet, p53 activates expression of the Pirh2-encoding RCHY1 gene, thus forming a negative regulatory feedback loop [1113]. Besides p53 and its homologs p63 and p73 [14-16], there are several other targets of Pirh2 that play roles in cell cycle regulation, apoptosis activation, DNA-damage response and tumor transformation, such as Chk2, p2 7 Kip1 and Pol $\eta$ [17-19]. Pirh2 ubiquitinates these proteins and directs them into the degradation pathway thus affecting apoptosis induction, cell cycle regulation and DNA repair. However the involvement of Pirh2 in these processes still 
needs further investigation.

Despite the negative effect on $\mathrm{p} 53$, the role of Pirh2 in cancer progression is rather obscure. For example, Duan et al. carried out the analysis of Pirh2 expression in human lung neoplasms paired with normal lung tissues. As the result, it was shown that expression of Pirh2 was increased in $27(84 \%)$ of 32 human specimens [20]. Similar results were obtained for Pirh2 expression in prostate cancer. Overexpression of Pirh2 was detected in 73 of $82(89 \%)$ resected human prostate cancer specimens [21]. Overexpression of Pirh2 in hepatocellular carcinoma (HCC) cells was found to correlate with vein invasion, TNM stage and number of tumor nodes [22]. Shimada and colleagues reported that in about $60 \%$ cases of human HNSCC increased Pirh2 levels were observed in comparison with $0 \%$ of normal mucosa [23]. These data strongly suggest that Pirh2 is an oncogene. On the other hand, genome-wide microarray studies showed that lower levels of Pirh 2 mRNA were associated with reduced survival of patients with breast and ovarian cancer, and lung squamous carcinomas [24]. Thus, the role of Pirh2 in tumorigenesis seems to be ambiguous and needs further investigation.

To elucidate the p53-independent role of Pirh2 in lung cancer we examined the effect of Pirh2 on proliferation, invasion potential and drug resistance of H1299 p53-negative lung carcinoma cells.

\section{RESULTS}

\section{Pirh2 affects proliferation of $\mathrm{H1299}$ cells}

To elucidate the role of Pirh2 in p53-negative cancer cells we decided to assess the effect of Pirh2 expression on classical characteristics of tumorigenecity: proliferation, invasion potential, and resistance to anti-cancer drugs. We chose H1299 cells since these lung carcinoma cells are negative for $\mathrm{p} 53$ and express relatively low levels of Pirh2 thus making these cells a convenient system to study effects of Pirh2 ectopic expression.

To generate H1299 cells with different status of Pirh2 we stably transduced these cells with lentiviral (LeGO and pLKO) vectors that express Pirh2 cDNA or specific shRNA against this gene, respectively. Cells with empty LeGO and pLKO expressing scrambled shRNA were used as appropriate controls. The efficiency of transduction was verified by FACs analysis as shown in Figure $1 \mathrm{~A}$. To evaluate the levels of Pirh2 either overexpression or down-regulation mediated by LeGOPirh2 and pLKO Pirh2 shRNA vectors, respectively, we used western blotting (Figure 1B). As most of E3 ubiquitin ligases Pirh2 undergoes auto-ubiquitination followed by proteasomal degradation. Therefore, to enhance the Pirh2

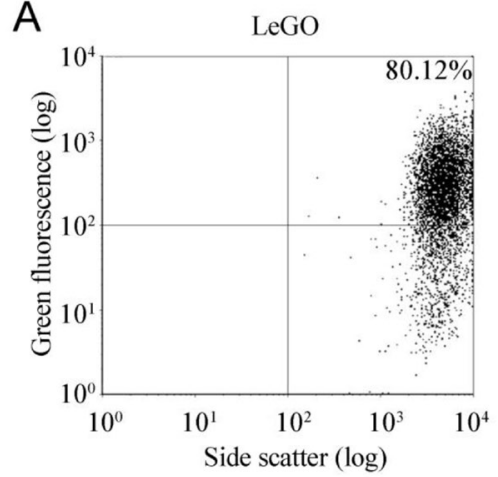

C

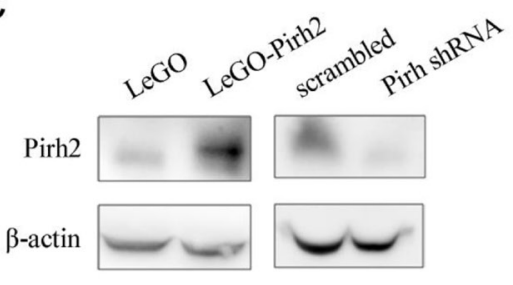

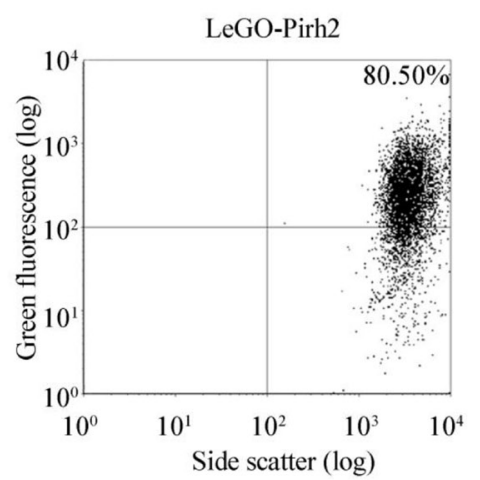

B

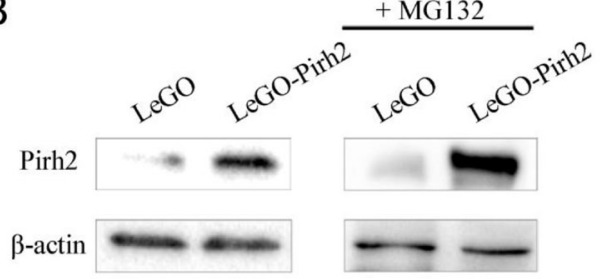

D

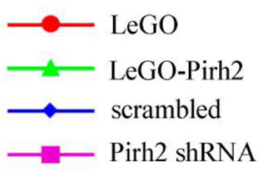

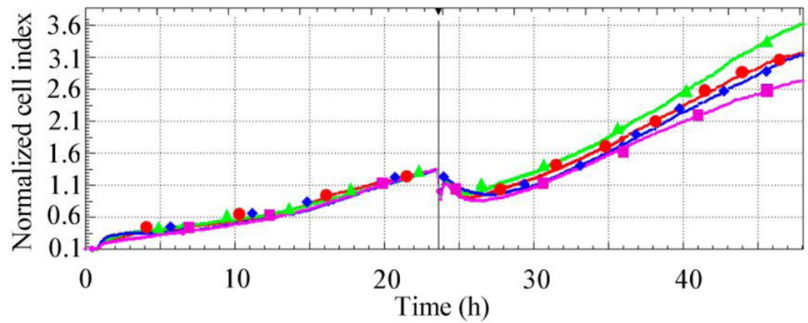

Figure 1: Pirh2 affects proliferation of H1299 cells. (A) Evaluation of transduction efficiency of H1299 cells with LeGO- and LeGO-Pirh2 by FACs analysis of GFP-positive cells. (B) Western blot analysis of Pirh2 protein levels in H1299 cells stably expressing LeGO-Pirh2 and LeGO control before (left panel) and after (right panel) the $16 \mathrm{~h}$ treatment with $5 \mu \mathrm{M}$ proteasome inhibitor MG132. (C) Western blot analysis of Pirh2 protein levels in H1299 cells stably expressing LeGO-Pirh2, LeGO, Pirh2 shRNA pLKO and scrambled shRNA pLKO vectors, respectively. (D) Proliferation rates of H1299 LeGO-Pirh2, control cell line H1299 LeGO, and H1299 Pirh2 shRNA cells. H1299 cells with scrambled shRNA were used as control. The data are shown as cell index graphs. 
western blot signal we treated stably transduced cells with the proteasome inhibitor, MG132. As shown in Figure 1B samples with stable overexpression of Pirh2 in H1299 cells was readily detected by Pirh2-specific antibody. MG132 treatment (right panel) further augmented the signal (Figure 1B). We also evaluated the efficacy of shRNA-mediated knockdown of Pirh2 by comparing Pirh2 western blot signals in control cells (scrambled shRNA) and cells with attenuated expression of Pirh2 (Pirh2 shRNA) (Figure 1C). We found that stable expression of Pirh2 shRNA construct effectively attenuated endogenous expression of Pirh2.

In order to measure the proliferation rate of H1299 cells with different status of Pirh2 we performed realtime monitoring of cell growth using the xCELLigence system (Figure 1D). This system (used hereafter) allows estimating cell index in real time - the parameter based on impedance measurement and reflecting the number of cells

A

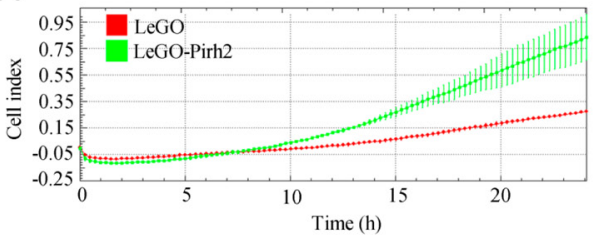

C

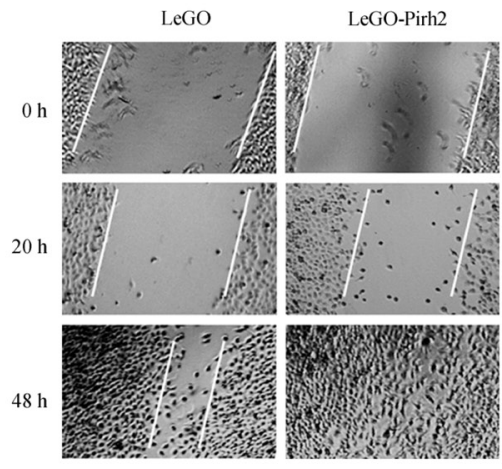

$E$

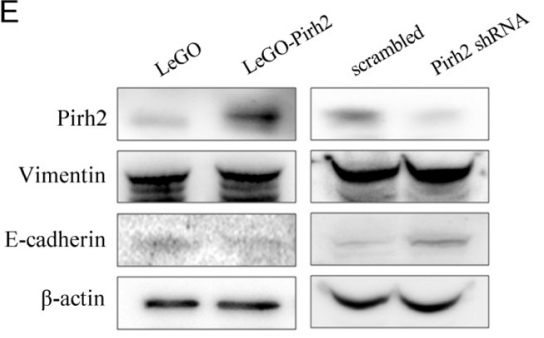

attached to the surface of the experimental chamber. Using this instrument, we showed that Pirh2 overexpression (LeGO-Pirh2) promoted cell proliferation while silencing of Pirh2 by shRNA (shRNA-Pirh2) attenuated the proliferative potential of H1299 cells (Figure 1D). Importantly, appropriate controls (LeGO and scrambled cells) exhibited very similar proliferation rates strongly suggesting that these effects were specific to Pirh2 and not to transduction manipulations.

\section{Pirh2 enhances the migratory potential of H1299 cells}

Next, we wanted to examine the effect of Pirh2 on migratory potential of H1299 cells. To address this question, we used the CIM-plate device of xCELLigence system. CIM-plate consists of two chambers separated by microporous membrane (pore size is $8 \mu \mathrm{m}$ ) attached

B

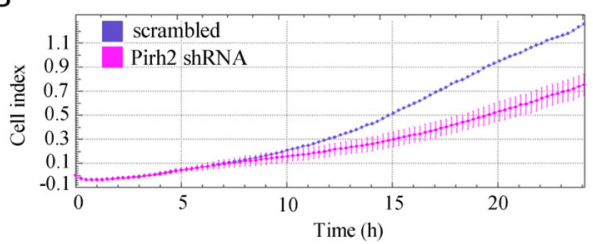

D

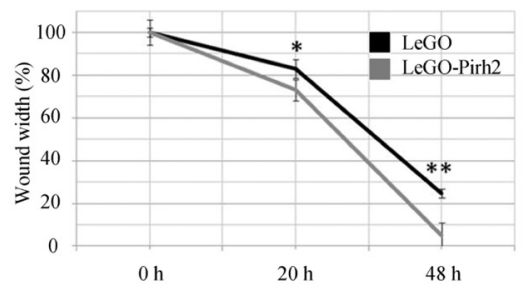

$\mathrm{F}$

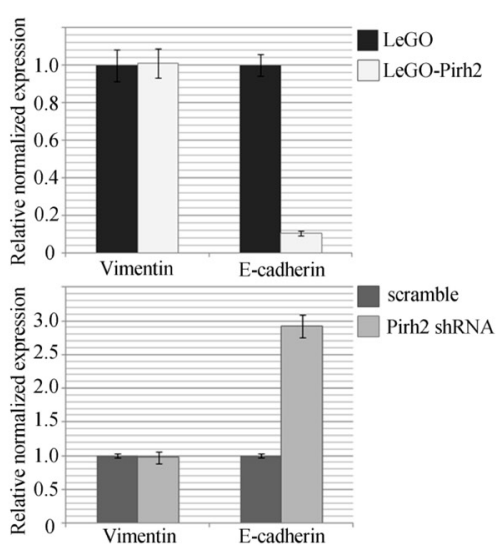

Figure 2: Pirh2 enhances the migratory potential of H1299 cells. (A) The migratory activity of H1299 LeGO-Pirh2 cells and control H1299 LeGO cells shown as cell index graphs. (B) The migratory activity of H1299 Pirh2 shRNA cells and control H1299 scrambled shRNA cells shown as cell index graphs. (C) The wound-healing assay using LeGO-Pirh2- and control LeGO-expressing H1299 cells. (D) Statistical results of wound-healing assay. Error bars indicate \pm SD. Student's $t$-test was performed for Pirh2-LEGO H1299 cells and their appropriate H1299 LEGO controls. * $\mathrm{P}<0.05$; ** $\mathrm{P}<0.01$. Protein (E) and mRNA (F) expression levels of vimentin and E-cadherin in LeGO-Pirh2, control LeGO, Pirh2 shRNA and control scrambled shRNA H1299 cells. 
to microelectrodes. In this case, cell index calculated on the base of impedance measurements reflects the amount of cells migrated through micropores. Our results showed that Pirh2 overexpression enhanced the migration rate compared to control H1299 cells (Figure 2A). On the contrary, silencing of Pirh2 by shRNA resulted in attenuation of H1299 cell migration (Figure 2B). The participation of Pirh2 in regulation of the migration ability of H1299 cells was also confirmed by woundhealing assay. We observed a $50 \%$ faster wound closure for LeGO-Pirh2-expressing cells when compared to the control LeGO cells (Figure 2C and 2D). These unexpected results prompted us to evaluate the effect of Pirh2 on the expression of established markers of EMT, vimentin and E-cadherin, which also regulate the migratory potential of cells (Figure 2E and 2D). According to our results, the protein level of E-cadherin is reduced in Pirh2overexpressing H1299 cells. Accordingly, E-cadherin was increased in Pirh2-deficient H1299 cells. Further, we showed that the effect of Pirh2 on E-cadherin occurs on the transcriptional level since mRNA expression of $\mathrm{CDH} 1$ was attenuated 5-fold upon overexpression of Pirh2 (Figure 2D, upper). In sharp contrast, the level of $\mathrm{CDH1}$ was increased 3-fold in the cells expressing Pirh2 shRNA compared to the cells with scrambled shRNA (Figure 2D, lower). Importantly, mRNA and protein expression levels of vimentin were unchanged, which argues that Pirh2 specifically affected E-cadherin.

\section{Pirh2 affects the resistance of $\mathrm{H} 1299$ cells to doxorubicin}

Finally, we assessed the impact of Pirh2 on drug resistance of H1299 cells, which is an important characteristic of curability and aggressiveness of cancer cells. Figure 3 shows the data of real-time proliferation after the treatment with doxorubicin of cell lines with overexpressed (LeGO-Pirh2) and silenced expression of Pirh2 (Pirh2 shRNA), and their controls (LeGO and scrambled, respectively). We observed that Pirh2 overexpression maintained cell proliferation while silencing of Pirh2 significantly reduced the proliferative potential of $\mathrm{H} 1299$ cells by 16 hours of treatment (Figure 3A). To confirm these results, we employed MTT assay on H1299 cells transfected with pcDNA or pcDNAPirh2 vectors (Figure 3D) treated with doxorubicin or cisplatin. We found that Pirh2 overexpression augmented the viability of $\mathrm{H} 1299$ cells treated with doxorubicin but did not affect their resistance to cisplatin (Figure 3B and 3C). These results suggest that Pirh2 selectively benefit cancer cells to resist genotoxic drugs that cause double strand DNA breaks, e.g. doxorubicin, but not the ones that induce cross-linking of DNA strands.

To substantiate our MTT results we also assessed the effect of Pirh2 on sensitivity of H1299 cells to doxorubicin by colony formation assay. To this end, we

A
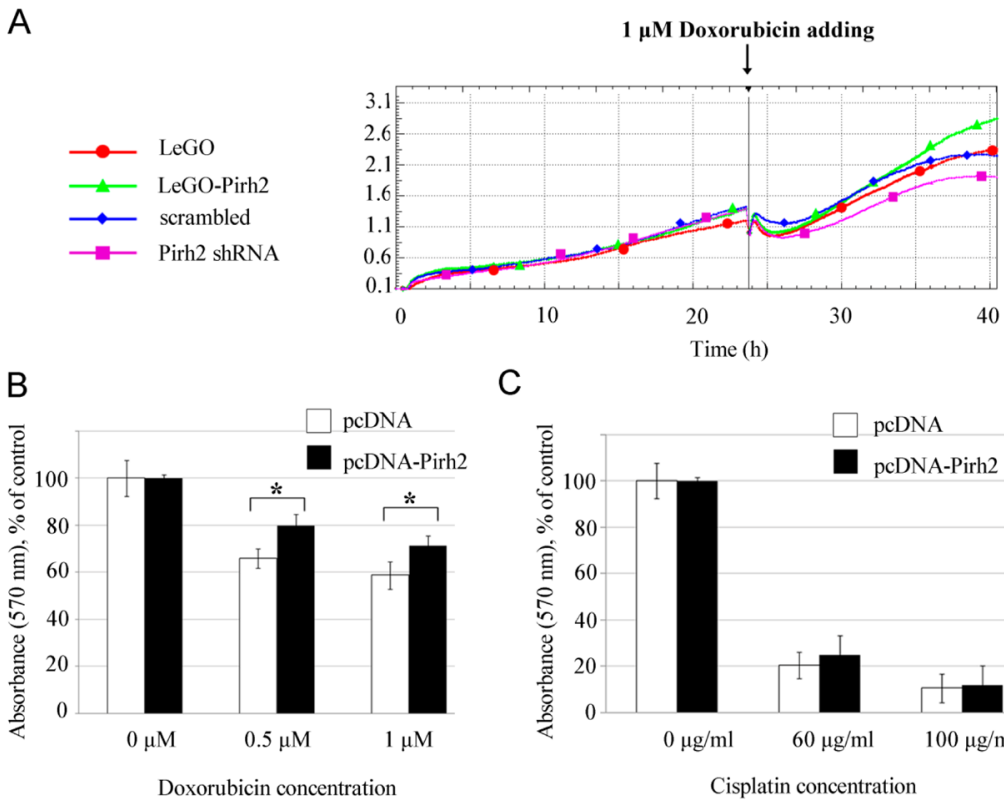

C

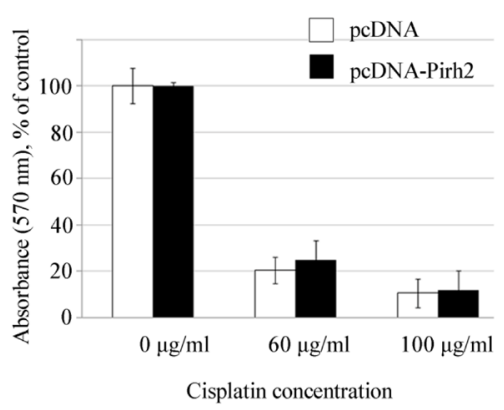

D

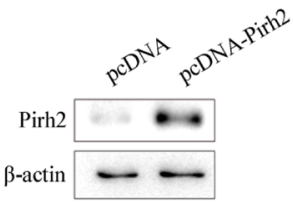

Figure 3: Pirh2 affects the resistance of H1299 cells to doxorubicin. (A) Cell index graphs obtained for H1299 cells stably expressing LeGO-Pirh2, control LeGO, Pirh2 shRNA, and scrambled shRNA, respectively, which were exposed to $1 \mu \mathrm{M}$ doxorubicin. Viability of $\mathrm{H} 1299$ cells transfected with the pcDNA-Pirh2 and control pcDNA plasmids after $24 \mathrm{~h}$ treatment with doxorubicin $(0,5 \mu \mathrm{M}$ and $1 \mu \mathrm{M})(\mathrm{B})$ or cisplatin $(60 \mu \mathrm{g} / \mathrm{ml}$ and $100 \mu \mathrm{g} / \mathrm{ml})(\mathrm{C})$ assessed by MTT assay. Error bars indicate \pm SD within quadruplicate. Student's $t$-test was performed for Pirh2 overexpressing samples and their appropriate controls. ${ }^{*} \mathrm{P}<0.05$. (D) Western blot analysis of Pirh2 protein levels in H1299 cells after transfection with pcDNA-Pirh2 or control pcDNA plasmids. 
compared pair-wise the numbers of colonies formed by control LeGO cells and cells overexpressing Pirh2 (LeGO-Pirh2), and cells expressing scrambled shRNA against H1299 cells with attenuated expression of Pirh2 (Pirh2 shRNA) (Figure 4A, B, and C). Overexpression of Pirh2 increased the number of colonies both in untreated and treated with doxorubicin cells (Figure 4A, left panel and 4B). On the contrary, attenuation of Pirh2 elicited an opposite effect (Figure 4A, right panel and 4C) by increasing the sensitivity of cells to doxorubicin compared to scramble shRNA-expressing cells. It should be noted that the difference in cytotoxicity between the cell line variants became noticeable only at $10 \mathrm{nM}$ concentration of doxorubicin. Yet, at the concentration of $25 \mathrm{nM}$ of doxorubicin the colony formation was almost completely suppressed in all the observed cell lines. Based on these results we estimated the ID50 concentration of doxorubicin for colony formation of H1299 LeGO cells $\sim 10-11 \mathrm{nM}$ and H1299 LeGO-Pirh2 cells $\sim 15-16$
nM. These results strongly suggest that Pirh2 promotes resistance of cancer cells to doxorubicin.

Furthermore, we determined the effect of Pirh2 on apoptosis in response to doxorubicin treatment. Analysis of Annexin V staining of H1299 cells with different levels of Pirh2 expression (scrambled versus shRNA-Pirh2) by FACS revealed the increased level of apoptosis in Pirh2deficient cells compared with control (Figure 4D) both before and after doxorubicin treatment. These results further confirm the contribution of Pirh2 to proliferation and resistance of H1299 cells to doxorubicin.

\section{Pirh2 enhances c-Myc expression, which correlates with poor survival of lung cancer patients}

To elucidate the potential molecular mechanism that underlies the tumorigenic properties of Pirh2 we examined

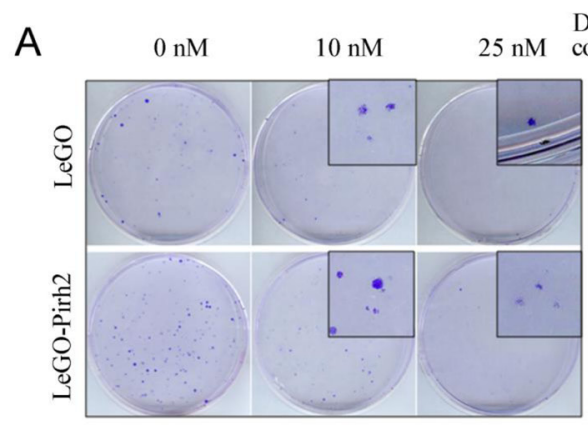

B

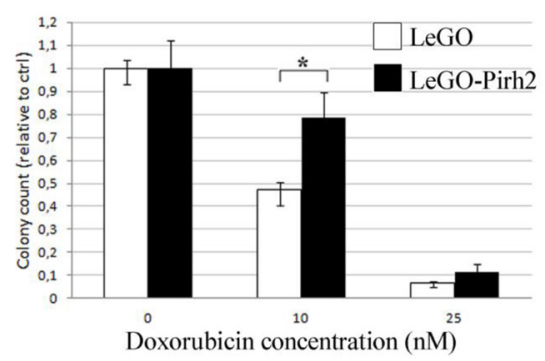

Doxorubicin

concentration $\quad 0 \mathrm{nM} \quad 10 \mathrm{nM} \quad 25 \mathrm{nM}$

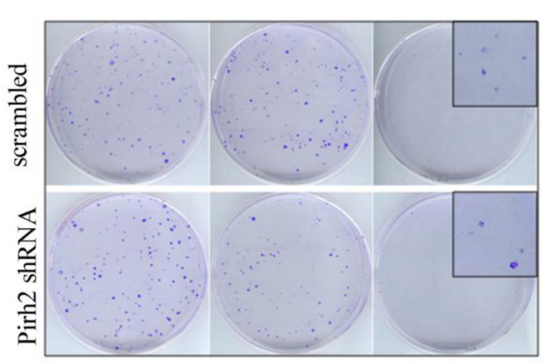

C

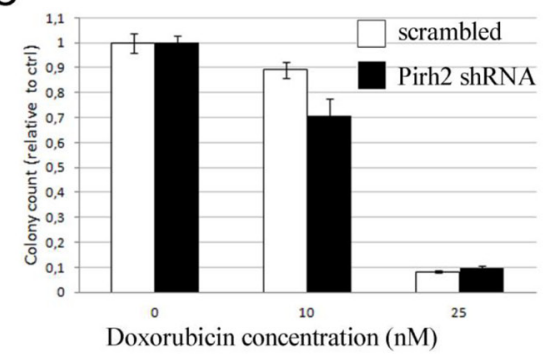

D
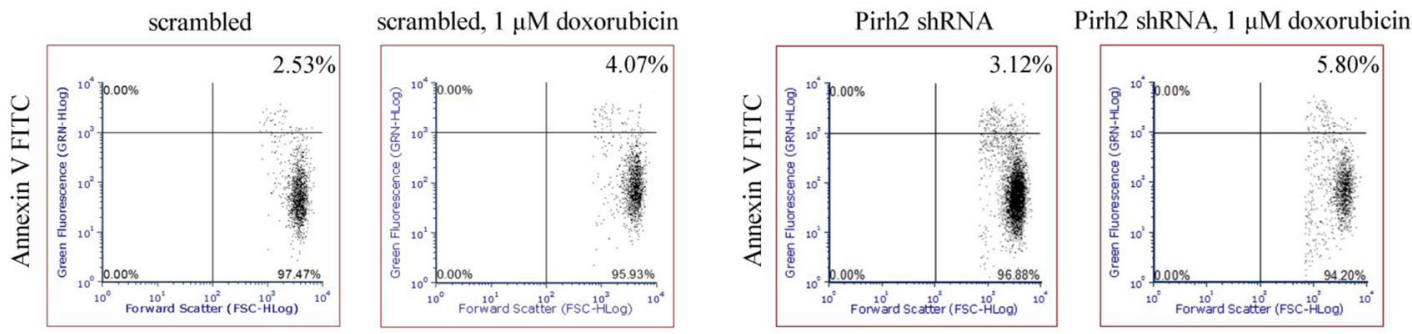

Figure 4: Effect of Pirh2 on colony formation and apoptosis of H1299 cells treated with doxorubicin. (A) Results of colony formation assay obtained for H1299 cells stably expressing LeGO-Pirh2, control LeGO, Pirh2 shRNA, and scrambled shRNA, both untreated and treated with $10 \mathrm{nM}$ and $25 \mathrm{nM}$ doxorubicine for 3 days. (B) Statistical analysis of the relative colonies numbers for LeGOPirh2 versus LeGO control and (C) Pirh2 shRNA versus scrambled shRNA was carried out. Error bars indicate \pm SEM within triplicate, $* \mathrm{P}<0.05$ (Student's $t$-test). (D) The apoptosis rate of Pirh2 shRNA, and scrambled shRNA H1299 cells untreated and treated with $1 \mu \mathrm{M}$ doxorubicin for 16 hours was determined by Annexin V-FACS analysis. 
expression levels of two the most critical masterregulators of proliferation and cell survival, NF- $\kappa \mathrm{B}$ and c-Myc. Control H1299 cells and cells stably expressing Pirh2 were subjected to western blotting analysis using NF- $\kappa$ B p65/RelA- and c-Myc-specific antibodies (Figure 5A). Surprisingly, we found that while p65/RelA expression levels were the same in control and LeGOPirh2 cells, the level of c-Myc protein was increased in cells overexpressing Pirh2 (Figure 5A). To get further insights into this observation we measured transcription of both p65/RelA and c-Myc genes as a function of Pirh2 overexpression. Interestingly, we noticed over 3-fold increase of c-Myc transcription in the presence of ectopic Pirh2 compared to control cells, whereas RelA levels were comparable in both types of cells (Figure 5B).

To corroborate these results in the biological setting we carried out the bioinformatics analysis to establish a correlation between the Pirh2 and c-Myc co-expression and survival of lung cancer patients using the Syntarget software as described previously [32]. Interestingly, the elevated expression of $R C H Y 1$ by itself correlated with better survival of patients with different tumors ([24] and data not shown). In contrast, as shown at Figure $5 \mathrm{C}$, high levels of co-expression of the Pirh2-coding RCHY1 gene and c-Myc correlated significantly with

A

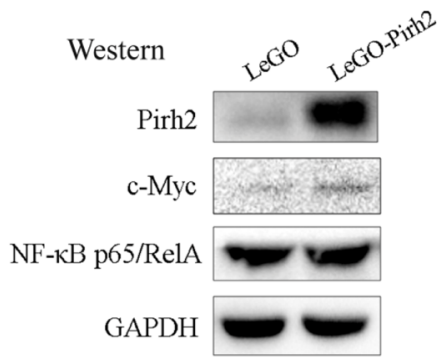

C

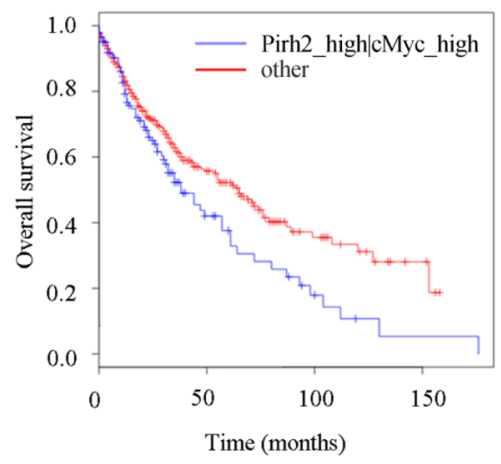

Lung squamous cell carcinoma (GEO ID: TCGA LUSC, P-value: 0,0488) poor survival outcome (Figure 5C). On the contrary, when the expression of both RCHY1 and c-Myc genes was low, survival outcomes of patients was significantly improved (Figure 5D). Thus, it can be hypothesized that one of the potential mechanisms by which Pirh2 increases proliferation, the migratory ability and drug resistance of H1299 cells may involve the augmented expression of c-Myc, which is known to regulate the aforementioned processes.

\section{DISCUSSION}

Recent studies have brought attention to Pirh2 as an E3 ubiquitin ligase that regulates cellular homeostasis upon genotoxic stress both in the p53-dependent and p53-independent cellular context. Several important targets of Pirh2 playing key roles in apoptosis, cell cycle progression and DNA repair have been identified. These include the p53 family proteins (p53, p63 and p73), Chk2, p2 $7^{\text {Kip1 }}$ and Pol $\eta[12,14-19]$. However, the role of Pirh2 in tumorigenesis is still controversial as expression of human Pirh2 in lung, ovarian, and breast cancers correlates with decreased patients' survival [24]. Both experimental and bioinformatics data presented in this study strongly suggest that Pirh2 functions as an oncogene in lung

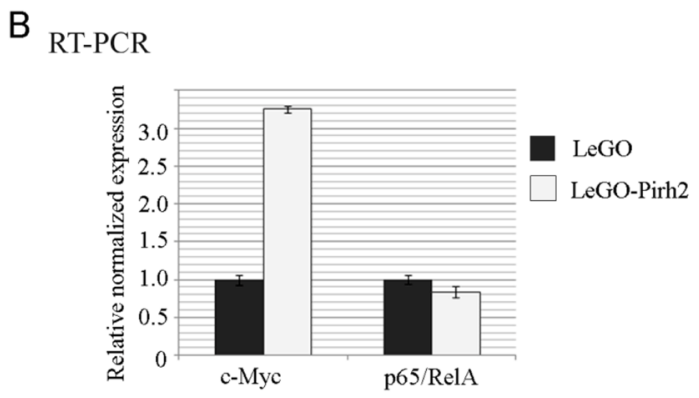

D

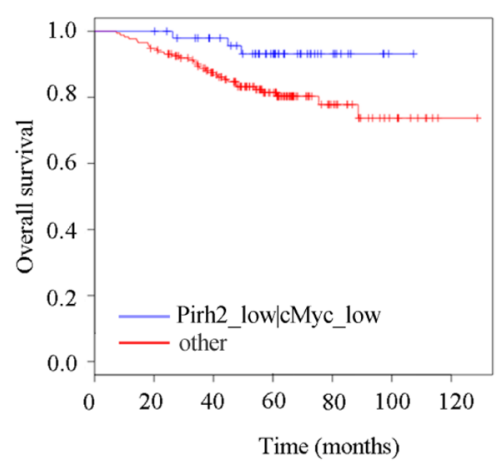

Lung adenocarcinoma stage I-II (GEO ID: GSE31210, P-value: 0,0289)

Figure 5: Pirh2 augments c-Myc expression and correlates with poor survival of lung cancer patients. Protein (A) and mRNA (B) expression levels of c-Myc and NF-kB p65/RelA in LeGO-Pirh2 and control LeGO H1299 cells. The bioinformatics analysis of synergistic effect of Pirh2 and c-Myc co-expression on (C) lung squamous cell carcinoma patients survival (GEO dataset ID: TCGA LUSC), and (D) lung adenocarcinoma stage I-II patients survival (GEO dataset ID: GSE31210), p-values are indicated. 
non-small cell carcinomas. Forced expression of Pirh2 increases the proliferative potential, drug resistance and migration ability of H1299 cells, which is in agreement with experimental data of other researchers. For example, Yuan et al. (2008) reported that Pirh2 silencing by siRNA resulted in reduction of cell proliferation and increased apoptosis in p53-positive A549 lung adenocarcinoma cells [26]. The authors proposed that these effects were caused by Pirh2-mediated degradation of p27 ${ }^{\mathrm{Kip} 1}$ cell cycle inhibitor. However, this speculation requires experimental confirmation. Furthermore, knockdown of Pirh2 by siRNA was reported to suppress the colony formation potential of H1299 cells [14]. Jung and colleagues also demonstrated that Pirh2 overexpression deceased the number of cells in sub-G1 stage after doxorubicin treatment indicating that Pirh2 attenuated the level of apoptosis [14].

Increased resistance to pharmacological treatments is an important feature of aggressive tumors. In this respect, our results showed that ectopically expressed Pirh2 conferred selective resistance to doxorubicin. Doxorubicin induces double-strand breaks in DNA by inhibiting the ligase activity of DNA topoisomerase II. On the contrary, cisplatin mostly makes intra-strand cross-links of purine bases thereby activating nucleotide excision repair (NER) [27]. Based on these finding we speculate that Pirh2 specifically promotes double-strand breaks DNA repair by yet not fully understood mechanism. In this respect, it should be noted that our biochemical screening for novel Pirh2-interacting proteins revealed several new partners of Pirh2 involved in double-strand breaks DNA repair by both homologous recombination and non-homologous end joining (data not published).

Finally, we showed that Pirh2 promoted migratory potential of H1299 cells. Cellular migration and invasion are critical for the formation of metastases. This process is preceded by epithelial-to-mesenchymal transition, which converts epithelial cells into mesenchymal by silencing E-cadherin and augmenting expression of vimentin. Our results demonstrated that ectopic expression of Pirh2 further attenuated cellular levels of E-cadherin, the protein responsible for tight junctions between cells, while keeping the level of vimentin intact. This decrease of E-cadherin expression was paralleled by increased migration of Pirh2-overexpressing cells. It is likely, that in addition to E-cadherin, Pirh2 down-regulates several other gene products that inhibit migration. However, this interesting possibility needs to be tested experimentally.

To get insights into the possible mechanisms by which Pirh2 facilitates proliferation and migration of H1299 cells, we focused on two major regulators of cell proliferation and migration: $c-\mathrm{Myc}$ and $\mathrm{NF}-\kappa \mathrm{B}$ [28-31]. While Pirh2 did not affect NF- $\kappa$ B expression, unexpectedly, we found that Pirh2 stabilized c-Myc (Figure 5A). Our results contrasted the previously published ones on Pirh2-mediated ubiquitin-dependent degradation of the c-Myc protein [24]. In addition, we demonstrated that ectopically expressed Pirh2 induced c-Myc transcription (Figure 5B). To corroborate these seemingly contradictory results one can assume that Pirh2 affects c-Myc expression indirectly and this effect strongly depends on a particular cellular context. As a possible explanation of Pirh2-mediated regulation of c-Myc, it can be speculated that it is mediated via the degradation of p73, which is known to repress c-Myc expression and yet it is an established target of Pirh2 [14, 32]. Therefore, attenuation of p73 by Pirh2 should augment the c-Myc expression. This interesting possibility will need experimental validation in the future. It should also be noted that the c-Myc protein was reported to suppress E-cadherin by activating E-cadherin-specific microRNA miR-9 that mediates attenuation of E-cadherin expression [33]. This fact is consistent with our results showing that Pirh2-mediated up-regulation of c-Myc was concomitant with E-cadherin repression (Figure $5 \mathrm{~A}$ and $2 \mathrm{E}$ ).

In summary, this study provides several lines of evidence that Pirh2 plays the oncogenic role in p53negative human non-small cell lung carcinoma cells by enhancing their proliferation, migration, and resistance to doxorubicin. Thus, our findings suggest that Pirh2 can be considered as a potential target for the development of anticancer therapies aimed at p53-negative tumors.

\section{MATERIALS AND METHODS}

\section{Plasmids}

The pcDNA-Pirh2 construct was obtained from S. Benchimol. For generating stable cell lines either overexpressing Pirh2 or a knockdown of Pirh2 lentiviral vectors LeGO-iG2 [34] and pLKO.1-TRC [35] were used, respectively. The following Pirhe-speicific shRNA oligonucleotides were annealed prior to cloning into the pLKO.1-TRC vector digested with Age1 and EcoR1 enzymes: sense5'-CCGGAATGTAACTTATGCCTAGCT ACTCGAGTAGCTAGGCATAAGTTACATTTTTTTG-3' and antisense 5'-AATTCAAAAAAATGTAACTTATGCC TAGCTACTCGAGTAGCTAGGCATAAGTTACATT-3'. Scrambled shRNA was used as a control. All obtained expression constructs were verified by sequencing. The lentiviral packaging plasmid psPAX2 and the envelope plasmid pMD2.G were a gift from Didier Trono (Addgene plasmids \# 12260 and \#12259).

\section{Manipulations with cells}

NCI-H1299 ATCC human non-small cell lung carcinoma cell line was cultured under standard conditions in RPMI 1640 medium (Thermo Fisher Scientific, CA, USA) supplemented with 10\% fetal bovine serum (FBS, Lonza, MD, USA), penicillin/streptomycin 
and $2 \mathrm{mM}$ L-glutamine. H1299 cells were transfected using X-tremeGENE HP reagent (Roche, Switzerland) according to the manufacturer's instructions. Stable cell lines were obtained using multiple rounds of transduction by lentiviral particles. Briefly, HEK293-T cells seeded on $10 \mathrm{~cm}$ plate $24 \mathrm{~h}$ before were transfected with plasmid mix (15 $\mu \mathrm{g}$ expression vector, $9.35 \mu \mathrm{g}$ psPAX2 and $5.32 \mu \mathrm{g}$ pMD2.G) by modified calcium phosphate transfection method [36]. The medium was changed $16 \mathrm{~h}$ after transfection and then lentiviral particles were collected every $24 \mathrm{~h}$. To concentrate lentiviral particles an ultracentrifugation procedure through sucrose cushion $(2 \mathrm{~h} 72.000 \mathrm{~g})$ was performed. The lentivirus titer was estimated on HEK293-T by limiting dilution assay. For the transduction procedure, 50,000 cells per well in full RPMI 1640 medium were seeded onto the 24-well plate. After $4 \mathrm{~h}$ Polybrene was added to each well to the final concentration $8 \mu \mathrm{g} / \mathrm{ml}$. Lentiviral particles were added to the cells with subsequent incubation for $24 \mathrm{~h}$. Four days after transduction the percentages of LeGO-iG2transduced cells were assessed by GFP expression using flow cytometer Guava EasyCyte 8 (EMD Millipore, MA, USA). The cells successfully transduced with pLKO.1TRC were subjected to puromycin selection $(0.5 \mu \mathrm{g} / \mathrm{ml})$ for 7 days. Pirh2 expression in all obtained cell lines was checked by Western blot analysis.

\section{Real-time cell proliferation and cell migration assays}

The tests were performed using xCELLigence system (ACEA Biosciences, CA, USA). For cell proliferation assays $2 \times 10^{4}$ cells were seeded in each well of E-plate 16 (ACEA Biosciences, CA, USA) in RPMI 1640 medium. Cell index was registered every 10 minutes. For cell migration assays $3 \times 10^{4}$ cells were seeded in each well of CIM-plate 16 (ACEA Biosciences, CA, USA) in full RPMI 1640 medium. Cell index was registered every 15 minutes.

\section{Wound healing (scratch) assay}

Cells were seeded in 12-well plates at $80 \%$ confluence in full RPMI 1640 medium. The next day linear scratches of the monolayer cell culture were created by $1000 \mu \mathrm{l}$ pipette tips. The scratch closure was observed and photographed at $0 \mathrm{~h}, 20 \mathrm{~h}$ and $48 \mathrm{~h}$ using TS100 inverted light microscope (Nikon, Japan).

\section{Colony formation assay}

The assay was performed essentially as described previously in [37]. Briefly, 500 cells were seeded at 10 $\mathrm{cm}$ culture plates in full RPMI 1640 medium for $24 \mathrm{~h}$ and then treated with $10 \mathrm{nM}$ and $25 \mathrm{nM}$ doxorubicin for 3 days. Then doxorubicin-containing medium was replaced with full RPMI 1640 medium and incubated for additional 7 days for colony formation. The colonies were fixed and stained for 10 minutes with fixing/staining solution containing $0.05 \%$ crystal violet, $1 \%$ formaldehyde, $1 \%$ methanol buffered with PBS. Following washes and drying the colonies were scored and analyzed manually. The experiments were performed in triplicates.

\section{MTT assay}

$24 \mathrm{~h}$ after transfection cells were seeded into 96-well plates at 3.000 cells per well in full RPMI 1640 medium for $24 \mathrm{~h}$ with subsequent $24 \mathrm{~h}$ treatment with doxorubicin $(0.5 \mu \mathrm{M}$ and $1 \mu \mathrm{M})$ and cisplatin $(60 \mu \mathrm{g} / \mathrm{ml}$ and $100 \mu \mathrm{g} /$ $\mathrm{ml}$ ). Then $20 \mu \mathrm{l} 5 \mathrm{mg} / \mathrm{ml}$ of Triazolyl Blue (MTT) solution was added to each well for $4 \mathrm{~h}$ at $37^{\circ} \mathrm{C}$. After removing MTT containing medium, $150 \mu \mathrm{l}$ isopropyl alcohol (acidified with $40 \mathrm{mM} \mathrm{HCl}$ ) was added to dissolve MTTformazan salt. The absorbance at $570 \mathrm{~nm}$ and $630 \mathrm{~nm}$ was measured using Pikon multiplate reader (Analytica, Russia).

\section{Annexin V staining.}

For Annexin $\mathrm{V}$ staining the FITC Annexin V Apoptosis Detection Kit (BD Pharmingen, CA, USA) was used according to the manufacture's instructions. Briefly, cells were washed with cold PBS and resuspended in the binding buffer provided by the manufacturer. $5 \mu \mathrm{l}$ of FITC Annexin V was added to $100 \mu \mathrm{l}$ of cell suspension and incubated for $20 \mathrm{~min}$. Analysis was performed using Guava EasyCyte 8 flow cytometer (Guava Technologies, Millipore, Billerica, MA, USA) (EMD Millipore, MA, USA).

\section{Quantitative PCR}

The standard procedure of total RNA extraction was performed using TRI Reagent (Sigma-Aldrich, MO, USA) according to the manufacturer's instructions. To eliminate traces of DNA total RNA was treated with DNase I (Thermo Fisher Scientific, CA, USA) according to the manufacturer's instructions and stopped with a standard phenol/chloroform extraction. The reverse transcription reaction with $2 \mu \mathrm{g}$ of total RNA was performed using RevertAid RT kit (Thermo Fisher Scientific, CA, USA). Quantitative Real Time PCR was carried out using SsoFast EvaGreen Master Mix (BioRad, CA, USA) in the BioRad CFX-96 real time system (BioRad, CA, USA). Relative expression was calculated using the $\Delta \Delta \mathrm{Ct}$ method. Sample $\mathrm{Ct}$ values were normalized to GAPDH. The oligonucleotides 
used for qPCR were as follows: GAPDH sense 5'-GAGGTCAATGAAGGGGTCAT-3' and antisense 5'-AGTCAACGGATTTGGTCGTA-3'; Vimentin sense 5'- TGTCCAAATCGATGTGGATGTTTC-3' and antisense 5'-TTGTACCATTCTTCTGCCTCCTG; E-cadherin sense 5'-CTTCTGCTGATCCTGTCTGATG-3' and antisense 5'-TGCTGTGAAGGGAGATGTATTG-3'; c-Myc sense 5'-CTCCTCCTCGTCGCAGTAGA-3' and antisense 5'-GCTGCTTAGACGCTGGATTT-3'; RelA/ p65 sense 5'-CGAATGGCTCGTCTGTAGTG-3' and antisense 5'-TGGTGGTATCTGTGCTCCTC-3'. All amplifications were performed in triplicates.

\section{Western blotting}

For western blot analysis whole-cell extracts were prepared. The primary antibodies against the analyzed proteins were used as follows: Pirh2 (1:1,000, EPR14980, Abcam, CA, USA); GAPDH (1:2,000, ab9484, Abcam, CA, USA); $\beta$-actin $(1: 5,000$, A3854, Sigma-Aldrich, MO, USA); NF-кB p65 (1:1,000, sc-8008, Santa Cruz, CA, USA); c-Myc (1:300, 9E10, Santa Cruz, CA, USA); Vimentin (1:5,000, RV202, BD Biosciences, CA, USA); E-cadherin (1:1,000, 36/E, BD Biosciences, CA, USA). Secondary antibodies used were anti-mouse and antirabbit (1:10,000; Sigma-Aldrich, MO, USA).

\section{Bioinformatics}

Correlations of expression levels of RCHY1 (Pirh2) alone and in combination with $m y c$ (c-Myc) with the survival rates of lung cancer patients were calculated by algorythms described by Amelio et al. [38]. Individual contributions of RCHY1 and MYC genes were examined as described in Antonov et al. [39, 40] using publically available Gene Expression Omnibus (GEO) microarray data.

\section{Statistical analysis}

Data are shown as mean \pm standard deviation (SD) or standard error of the mean (SEM) of at least three replicates. Statistical significance was analyzed using Student's t-test. $\mathrm{P}<0.05$ was considered significant. $\mathrm{P}<0.05$ is denoted as *, $\mathrm{P}<0.01$ as $* *$.

\section{ABBREVIATIONS}

ATCC, American Type Culture Collection; Chk2, checkpoint kinase 2; EMT, epithelial-to-mesenchymal transition; GFP, green fluorescent protein; HCC, hepatocellular carcimoma; TNM, Tumor-Node-Metastasis classification of malignant tumours; HNSCC, head and neck squamous cell carcinoma; NFאB, nuclear factor- kappa B; Poln, DNA polymerase eta; RCHY1, RING finger and $\mathrm{CHY}$ zinc finger domain-containing protein 1.

\section{ACKNOWLEDGEMENTS}

The authors are grateful to S. Benchimol (University of Toronto, Canada) and X. Chen (University of California at Davis, US) for generous sharing with various reagents. This study was supported by in part, by RFBR research projects No.16-34-00869 mol_a and No.16-34-60228 mol_a_dk. The bioinformatics analysis was supported by the Molecular and Cellular Biology Program from MCB RAS to A.A. and N.A.B. All experiments on cell index measurements were supported by a grant from the Russian Science Foundation 14-50-00068.

\section{CONFLICTS OF INTERESTS}

The authors declare no conflict of interests.

\section{REFERENCES}

1. Beitel L, Elhaji Y, Lumbroso R, Wing S, Panet-Raymond V, Gottlieb B, Pinsky L and Trifiro M. Cloning and characterization of an androgen receptor N-terminalinteracting protein with ubiquitin-protein ligase activity. Journal of molecular endocrinology. 2002; 29(1):41-60.

2. Daks A, Melino D and Barlev N. The role of different E3 ubiquitin ligases in regulation of the P53 tumor suppressor protein. Tsitologiia. 2012; 55(10):673-687.

3. Wang Z, Yang B, Dong L, Peng B, He X and Liu W. A novel oncoprotein Pirh2: rising from the shadow of MDM2. Cancer science. 2011; 102(5):909-917.

4. Barlev N, Sayan B, Candi E and Okorokov A. The microRNA and p53 families join forces against cancer. Cell death and differentiation. 2010; 17(2):373.

5. Suzuki K and Matsubara H. Recent advances in p53 research and cancer treatment. BioMed Research International. 2011; 2011.

6. Grigoreva TA, Tribulovich VG, Garabadzhiu AV, Melino G and Barlev NA. The $26 \mathrm{~S}$ proteasome is a multifaceted target for anti-cancer therapies. Oncotarget. 2015; 6(28):24733.

7. Beckerman $\mathrm{R}$ and Prives $\mathrm{C}$. Transcriptional regulation by p53. Cold Spring Harbor perspectives in biology. 2010; 2(8):a000935.

8. Morgunkova A and Barlev NA. Lysine methylation goes global. Cell cycle. 2006; 5(12):1308-1312.

9. Moiseeva TN, Bottrill A, Melino G and Barlev NA. DNA damage-induced ubiquitylation of proteasome controls its proteolytic activity. Oncotarget. 2013; 4(9):1338-1348.

10. Tai E. (2010). Characterization of the E3 Ubiquitin Ligase Pirh2. University of Toronto).

11. Feng Z, Hu W, de Stanchina E, Teresky AK, Jin S, Lowe $\mathrm{S}$ and Levine AJ. The regulation of AMPK $\beta 1$, TSC2, and 
PTEN expression by p53: stress, cell and tissue specificity, and the role of these gene products in modulating the IGF-1-AKT-mTOR pathways. Cancer research. 2007; 67(7):3043-3053.

12. Leng RP, Lin Y, Ma W, Wu H, Lemmers B, Chung S, Parant JM, Lozano G, Hakem R and Benchimol S. Pirh2, a p53induced ubiquitin-protein ligase, promotes p53 degradation. Cell. 2003; 112(6):779-791.

13. Sheng Y, Laister RC, Lemak A, Wu B, Tai E, Duan S, Lukin J, Sunnerhagen M, Srisailam S and Karra M. Molecular basis of Pirh2-mediated p53 ubiquitylation. Nature structural \& molecular biology. 2008; 15(12):1334-1342.

14. Jung Y-S, Qian Y and Chen X. The p73 tumor suppressor is targeted by Pirh2 RING finger E3 ubiquitin ligase for the proteasome-dependent degradation. Journal of Biological Chemistry. 2011; 286(41):35388-35395.

15. Jung Y-S, Qian Y, Yan W and Chen X. Pirh2 E3 ubiquitin ligase modulates keratinocyte differentiation through p63. Journal of Investigative Dermatology. 2013; 133(5):11781187.

16. Wu H, Abou ZR, Flores ER and Leng RP. Pirh2, a ubiquitin E3 ligase, inhibits p73 transcriptional activity by promoting its ubiquitination. Molecular Cancer Research. 2011; 9(12):1780-1790.

17. Bohgaki M, Hakem A, Halaby M, Bohgaki T, Li Q, Bissey P, Shloush J, Kislinger T, Sanchez O and Sheng Y. The E3 ligase PIRH2 polyubiquitylates $\mathrm{CHK} 2$ and regulates its turnover. Cell Death \& Differentiation. 2013; 20(6):812822 .

18. Hattori T, Isobe T, Abe K, Kikuchi H, Kitagawa K, Oda T, Uchida C and Kitagawa M. Pirh2 promotes ubiquitindependent degradation of the cyclin-dependent kinase inhibitor p27Kip1. Cancer research. 2007; 67(22):1078910795.

19. Jung Y-S, Liu G and Chen X. Pirh2 E3 ubiquitin ligase targets DNA polymerase eta for $20 \mathrm{~S}$ proteasomal degradation. Molecular and cellular biology. 2010; 30(4):1041-1048.

20. Duan W, Gao L, Druhan LJ, Zhu W-G, Morrison C, Otterson GA and Villalona-Calero MA. Expression of Pirh2, a newly identified ubiquitin protein ligase, in lung cancer. Journal of the National Cancer Institute. 2004; 96(22):1718-1721.

21. Logan IR, Gaughan L, McCracken SR, Sapountzi V, Leung $\mathrm{HY}$ and Robson CN. Human PIRH2 enhances androgen receptor signaling through inhibition of histone deacetylase 1 and is overexpressed in prostate cancer. Molecular and cellular biology. 2006; 26(17):6502-6510.

22. Wang XM, Yang LY, Guo L, Fan C and Wu F. p53-induced RING-H2 protein, a novel marker for poor survival in hepatocellular carcinoma after hepatic resection. Cancer. 2009; 115(19):4554-4563.

23. Shimada M, Kitagawa K, Dobashi Y, Isobe T, Hattori T, Uchida C, Abe K, Kotake Y, Oda T and Suzuki H. High expression of Pirh2, an E3 ligase for p27, is associated with low expression of $\mathrm{p} 27$ and poor prognosis in head and neck cancers. Cancer science. 2009; 100(5):866-872.

24. Hakem A, Bohgaki M, Lemmers B, Tai E, Salmena L, Matysiak-Zablocki E, Jung Y-S, Karaskova J, Kaustov L and Duan S. Role of Pirh2 in mediating the regulation of p53 and c-Myc. PLoS Genet. 2011; 7(11):e1002360.

25. Lezina L, Aksenova V, Ivanova T, Purmessur N, Antonov A, Tentler D, Fedorova O, Garabadgiu A, Talianidis I and Melino G. KMTase Set7/9 is a critical regulator of E2F1 activity upon genotoxic stress. Cell Death \& Differentiation. 2014; 21(12):1889-1899.

26. Yuan S, Liping Z and Yang J. Effect of Pirh2 shRNA on Chemosensitivity of Lung Adencarcinoma Cell Line A549 to Cisplatin. Acta Medicinae Universitatis Scientiae et Technologiae Huazhong. 2008; 2:010.

27. Furuta T, Ueda T, Aune G, Sarasin A, Kraemer KH and Pommier Y. Transcription-coupled nucleotide excision repair as a determinant of cisplatin sensitivity of human cells. Cancer research. 2002; 62(17):4899-4902.

28. Higgins KA, Perez JR, Coleman TA, Dorshkind K, McComas WA, Sarmiento UM, Rosen CA and Narayanan R. Antisense inhibition of the p65 subunit of NF-kappa $\mathrm{B}$ blocks tumorigenicity and causes tumor regression. Proceedings of the National Academy of Sciences. 1993; 90(21):9901-9905.

29. Karin M. Nuclear factor- $\kappa \mathrm{B}$ in cancer development and progression. Nature. 2006; 441(7092):431-436.

30. Schmidt EV. The role of c-myc in cellular growth control. Oncogene. 1999; 18(19).

31. Waikel RL, Kawachi Y, Waikel PA, Wang X-J and Roop DR. Deregulated expression of c-Myc depletes epidermal stem cells. Nature genetics. 2001; 28(2):165-168.

32. Kartasheva NN, Lenz-Bauer C, Hartmann O, Schäfer H, Eilers $\mathrm{M}$ and Dobbelstein M. $\triangle \mathrm{Np} 73$ can modulate the expression of various genes in a p53-independent fashion. Oncogene. 2003; 22(51):8246-8254.

33. Ma L, Young J, Prabhala H, Pan E, Mestdagh P, Muth D, Teruya-Feldstein J, Reinhardt F, Onder TT and Valastyan S. miR-9, a MYC/MYCN-activated microRNA, regulates E-cadherin and cancer metastasis. Nature cell biology. 2010; 12(3):247-256.

34. Weber K, Bartsch U, Stocking C and Fehse B. A multicolor panel of novel lentiviral "gene ontology"(LeGO) vectors for functional gene analysis. Molecular Therapy. 2008; 16(4):698-706.

35. Moffat J, Grueneberg DA, Yang X, Kim SY, Kloepfer AM, Hinkle G, Piqani B, Eisenhaure TM, Luo B and Grenier JK. A lentiviral RNAi library for human and mouse genes applied to an arrayed viral high-content screen. Cell. 2006; 124(6):1283-1298.

36. Jordan $\mathrm{M}$ and Wurm F. Transfection of adherent and suspended cells by calcium phosphate. Methods. 2004; 33(2):136-143. 
37. Lezina L, Aksenova V, Fedorova O, Malikova D, Shuvalov O, Antonov AV, Tentler D, Garabadgiu AV, Melino G and Barlev NA. KMT Set7/9 affects genotoxic stress response via the Mdm2 axis. Oncotarget. 2015; 6(28):25843.

38. Amelio I, Tsvetkov P, Knight R, Lisitsa A, Melino G and Antonov A. SynTarget: an online tool to test the synergetic effect of genes on survival outcome in cancer. Cell Death \& Differentiation. 2016.

39. Antonov A, Krestyaninova M, Knight R, Rodchenkov I, Melino G and Barlev N. PPISURV: a novel bioinformatics tool for uncovering the hidden role of specific genes in cancer survival outcome. Oncogene. 2014; 33(13):16211628.

40. Antonov AV. BioProfiling. de: analytical web portal for high-throughput cell biology. Nucleic acids research. 2011:gkr372. 